\title{
Patient considerations in the use of tapentadol for moderate to severe pain
}

\author{
This article was published in the following Dove Press journal: \\ Drug, Healthcare and Patient Safety \\ 2 July 2013 \\ Number of times this article has been viewed
}

\section{Nalini Vadivelu \\ Yili Huang \\ Brian Mirante \\ Michael Jacoby \\ Ferne R Braveman \\ Roberta L Hines \\ Raymond Sinatra}

Department of Anesthesiology, Yale University, New Haven, CT, USA
Correspondence: Nalini Vadivelu Department of Anesthesiology, Yale University, 333 Cedar Street, New Haven, CT, USA 06520

Tel +l 2037842802

Fax +I 2037856664

Email nalini.vadivelu@yale.edu
Abstract: Poorly controlled acute and chronic pain can increase morbidity, impair quality of life and prolong disability. Over 80 percent of post surgical patients report moderate to severe uncontrolled postoperative pain. Over-reliance on potent opioid agonists can lead to several opioid related side effects such as gastrointestinal intolerability, respiratory depression and cognitive impairment. A recently approved dual acting central analgesic tapentadol may offer improved tolerability over traditional opioid agonists while having multimodal opioid and nonopioid analgesic benefits. Tapentadol, classified by the US Food and Drug Administration as a class 2 opioid, is currently marketed in the United States as immediate release (IR) NUCYNTA ${ }^{\circledR}$ for moderate to severe acute pain in tablets of $50 \mathrm{mg}, 75 \mathrm{mg}$, and $100 \mathrm{mg}$, and as extended release (ER) NUCYNTA ER ${ }^{\circledR}$ for the treatment of chronic moderate to severe pain in tablets of $50 \mathrm{mg}$, $100 \mathrm{mg}, 150 \mathrm{mg}, 200 \mathrm{mg}$, and $250 \mathrm{mg}$. Tapentadol is a low affinity mu opioid receptor agonist and a norepinephrine reuptake inhibitor. Tapentadol has no active metabolites and this property makes it useful in patients with hepatic and renal failure. Clinical trials with tapentadol IR showed that there was improved gastrointestinal tolerability and similar pain relief as compared to oxycodone IR. Tapentadol ER allows for twice daily dosing. Clinical trials showed that tapentadol ER could effectively relieve moderate to severe chronic pain and was associated with significantly fewer gastrointestinal adverse effects as compared to oxycodone controlled release. Tapentadol ER is indicated and has Food and Drug Administration approval for the treatment of chronic painful diabetic neuropathy. The most common side effects of tapentadol are nausea (30\%), vomiting (18\%), dizziness (24\%), and somnolence (15\%). Tapentadol, due to its potential synergistic effects on norepinephrine levels, is contraindicated in patients who have taken monoamine oxidase inhibitors within the last 14 days. Caution has to be exercised with the use of tapentadol IR and tapentadol ER in the presence of other central nervous system depressants such as neuroleptics, opioids, illicit drugs, muscle relaxants, sedatives, and anxiolytics. Keywords: tapentadol immediate release, tapentadol extended release, diabetic neuropathy, acute pain, chronic pain

\section{Introduction}

Acute and chronic pain are health issues that affect as many as $20 \%-30 \%$ of Americans and are among the most frequent reasons for physician visits and a leading cause of increased medical costs. In addition to a humanitarian desire to reduce suffering, poorly controlled pain can increase morbidity, delay recovery, impair quality of life, and result in prolonged disability. Out of over 70 million surgeries being performed yearly, ${ }^{1}$ more than $80 \%$ of patients report moderate to severe postoperative pain. ${ }^{2}$ This is important since we now recognize that poorly controlled acute pain can lead 
to prolonged convalescence and to the development of persistent pain states. ${ }^{3}$

A major factor leading to suboptimal pain management is overreliance on potent opioid agonists and associated dose-dependent adverse events. Opioid-related side effects are often dose dependent and include gastrointestinal (GI) intolerability such as nausea, vomiting, as well as other troublesome symptoms including cognitive impairment, physical and psychological dependence, and respiratory depression. In an effort to minimize dose dependent adverse events, many clinicians utilize a multimodal analgesic approach, employing nonopioid analgesics and adjuvants to reduce total opioid burden while still maintaining effective analgesia. $^{4}$

Opioid plus acetaminophen compounds, and oxycodone plus ibuprofen compounds (Combinox ${ }^{\circledR}$, Forest Laboratories Inc., Jersey City, NJ, USA) represent traditional multimodal approaches developed to potentiate opioid-based analgesia. Novel dual-acting analgesics such as tramadol and the more recently approved analgesic, tapentadol, provide multimodal opioid plus nonopioid analgesic benefits in a single molecule, and may offer improved tolerability than that observed with traditional opioid agonists.

\section{Opioids}

Opioids remain the pharmacological foundation of acute and chronic pain management; however, nonsteroidal anti-inflammatories, acetaminophen, and antidepressants represent important multimodal adjuncts. ${ }^{5}$ Despite their effectiveness as analgesics, opioids are commonly associated with significant adverse events that can be annoying and occasionally even life threatening. GI adverse events in patients with chronic pain - particularly nausea and vomiting in postsurgical settings, as well as severe constipation - are often intolerable and complicate clinical care. Opioidinduced nausea and vomiting is related to binding and activation of neurons in the chemoreceptor trigger zone. ${ }^{6}$ In some patients, GI events can be so frequent and/or severe that they negatively influence the success of analgesic therapy. Many patients choose less effective pain relief by either limiting or outright discontinuing opioid dosing. GI adverse events and pain related to opioid intolerance are responsible for ongoing visits to primary caregivers, pain clinics, or admissions to the emergency room, all resulting in an increased cost of care. Unfortunately, many patients suffer in silence rather than alerting the surgical office, or they avoid "offending" their caregivers with complaints related to analgesic choice and dosing regimen. ${ }^{7,8}$ Traditional opioid agonists suppress pain transmission by activating mu receptors in the spinal cord and brain; however, they also bind and activate receptors in the GI tract that mediate decreased bowel motility leading to postoperative ileus and opioid-induced bowel dysfunction. ${ }^{9}$

Other than reformulations, there have been no additions to the opioid family of analgesics for over a quarter of a century, until recently. Tapentadol is a potent analgesic developed by Grünenthal in Germany (Grünenthal GmbH, Aachen, Germany) along with Johnson and Johnson Pharmaceutical Research and Development (Johnson and Johnson Pharmaceutical Research and Development, LLC, San Diego, CA, USA) that provides two distinct mechanisms of activity in a single molecule. This profile has set it apart from pure opioid agonists, and represents the first in a new line of potent dual-acting, central analgesics.

Tapentadol is currently marketed in the United States under the brand name of NUCYNTA ${ }^{\circledR}$ (Janssen Pharmaceuticals, Inc., Titusville, NJ, USA) as immediate release (IR) $50 \mathrm{mg}, 75 \mathrm{mg}$, and $100 \mathrm{mg}$ oral tablets primarily indicated for moderate to severe acute pain, and NUCYNTA ${ }^{\circledR}$ extended release (ER) as $50 \mathrm{mg}, 100 \mathrm{mg}, 150 \mathrm{mg}, 200 \mathrm{mg}$, and $250 \mathrm{mg}$ ER tablets for moderate to severe chronic pain. The Food and Drug Administration (FDA) has classified tapentadol as a class 2 opioid; therefore, all the precautions that occur when prescribing these types of drugs must be adhered to. ${ }^{10}$

\section{Pain mechanisms and pharmacology of tapentadol}

Tapentadol is a dual-acting central analgesic that has lower affinity for mu opioid receptors (MOR) than traditional agonists such as morphine and oxycodone. In preclinical studies in rat and mouse models of acute and chronic pain, tapentadol was found to have 18 times lower binding affinity to MOR than morphine. Tapentadol, despite having lower affinity, provides highly effective analgesia with the ratio of the tapentadol to morphine-equivalent analgesic dose being 2.5:1. For example, the equianalgesic dose of oral morphine for a patient taking $100 \mathrm{mg}$ of oral tapentadol would be $40 \mathrm{mg}$. ${ }^{11}$

The mechanisms responsible for this overall gain in analgesic potency are related to the fact that tapentadol is a fairly selective, rapid-acting norepinephrine (NE) reuptake inhibitor. It provides additional pain suppression by increasing NE and activating alpha-2-adrenergic receptors in the spinal cord and brain. This dual analgesic effect of MOR activation and NE-reuptake inhibition provides analgesic effects equivalent to equipotent doses of oxycodone. In animal studies, tapentadol administration was associated 
with a large increase in extracellular NE, whereas serotonin levels increased minimally. ${ }^{12}$ Animal studies have shown that naloxone, a MOR antagonist, partially inhibits tapentadolmediated analgesia; however, the addition of the alpha-2antagonist, yohimbine, eliminated all analgesic effects. ${ }^{13}$ The 5-HT2-inhibitor ritanserin showed no effect on the analgesia provided by tapentadol. ${ }^{14}$

To fully understand the unique analgesic benefits of tapentadol, it is important to appreciate its interaction with ascending pathways responsible for pain perception and descending tracts that suppress noxious transmission. The ascending pathway is primarily excitatory. Noxious stimuli are received by second order dorsal horn neurons and projected via the ascending spinothalamic tract to regions of the midbrain, brainstem, thalamus, and cerebral cortex, including the limbic system. ${ }^{15}$ Activation of pre- and postsynaptic opioid receptors in the ascending pathway inhibits presynaptic release of noxious transmitters as well as postsynaptic depolarization of second-order cells. The descending pathway is primarily inhibitory. Neurons in the periaqueductal grey and rostral ventromedial medulla respond to ascending noxious stimuli and send inhibitory fibers to the dorsal horn. These fibers release NE and serotonin, which activate postsynaptic alpha-2 receptors and modulate noxious transmission. ${ }^{16}$

\section{Pharmacokinetic profile}

Tapentadol has no active metabolites. Many opioids are metabolized into active substances, which increase tapentadol's adverse effects and make its actions unpredictable. Morphine for example, is conjugated into morphine-3-glucoronide and morphine-6-glucoronide. Morphine-6-glucoronide is 100 times more potent than morphine on the MOR, and if accumulated (ie, in renal failure) contributes to prolonged respiratory depression. ${ }^{17}$

Tramadol is an earlier developed dual-acting analgesic that has several deficiencies not associated with tapentadol. First, tramadol is a prodrug, and in order for it to be effective, it must be metabolized by the cytochrome $\mathrm{P} 450$ system into its pharmacologically active metabolite, O-desmethyltramadol. There are no active metabolites of tapentadol.

Tapentadol is metabolized through the CYP2C9 and CYP2C19 system to N-desmethyltapentadol, and through uridine diphosphate glucuronyl transferase to tapentadolO-glucuronide. Both of these metabolites are inactive and have no analgesic activity. These qualities make tapentadol a more predicable analgesic as it avoids drug interaction uncertainties as well as safety and efficacy issues in patients with low or high CYP-2D6 enzyme expression. Tapentadol-
O-glucuronide is the major metabolite of tapentadol, and this is devoid of activity at the opioid receptors. About $99 \%$ of tapentadol IR and its metabolites are excreted in the kidneys. Tapentadol's analgesic effects are potentially more predictable than its predecessors, making it a useful analgesic in patients with renal failure and hepatic failure. ${ }^{18}$

\section{Tapentadol IR for moderate to severe acute pain}

Tapentadol was initially developed as an analgesic for acute surgical and nonsurgical pain. Tapentadol is currently approved to treat moderate to severe acute pain in an IR form and chronic moderate to severe pain in an ER form in patients over 18 years of age. ${ }^{19}$ It is effective against inflammatory, visceral, nociceptive, and neuropathic conditions. ${ }^{20}$

\section{Effective analgesia}

The efficacy of tapentadol IR as an analgesic as compared to placebo was demonstrated in one Phase II and two Phase III postsurgical trials. These trials were large, randomized, multicenter, double blinded, and placebo controlled. The Phase II trials evaluated a single dosing of tapentadol IR following dental procedures, whereas the Phase III pivotal trials were performed in patients recovering from bunionectomy.

The efficacy of tapentadol IR over placebo was shown in two separate 3-day Phase III trial with multidosing assessment in bunionectomy patients. In Bunionectomy Trial 1, tapentadol IR in doses of 50-75 and $100 \mathrm{mg}$ were superior to placebo and comparable to $15 \mathrm{mg}$ IR oxycodone. ${ }^{21}$ In Bunionectomy Trial 2, tapentadol IR doses of $50 \mathrm{mg}$ and $75 \mathrm{mg}$ were found to be superior to placebo and comparable to $10 \mathrm{mg}$ oxycodone IR. ${ }^{22}$

In addition after the first metatarsal bunionectomy with osteotomy, fewer patients treated with tapentadol IR required second-line rescue medications compared to those treated with oxycodone or placebo. ${ }^{23}$

\section{Cost with use of tapentadol IR}

An important patient consideration is cost. Tapentadol IR is a new drug, being marketed as NUCYNTA ${ }^{\circledR}$, and is more expensive than oxycodone, hydromorphone, or morphine. It is unclear whether reductions in the need to treat nausea and vomiting would outweigh the increased cost of the drug in most patients; however, it may be considered a drug of choice in patients presenting with histories of severe nausea and vomiting following treatment with traditional opioid agonists. When prescribing it, caution must be taken to weigh 
the risks and benefits. Studies have shown that when using tapentadol IR, reduced adverse effects as compared to other opioids offset the cost, and that patients are more likely to continue their regimen of tapentadol without switching to another opioid.

\section{Dosing tapentadol IR}

The starting dose of tapentadol IR in opioids-naïve patients is $50-75 \mathrm{mg}$. The $100 \mathrm{mg}$ dose may be more effective for patients with some opioid tolerance or for severe pain. The IR form can be used as 50-100 mg every 4-6 hours as needed for pain, and on the first day of treatment up to $700 \mathrm{mg} /$ day. From the second day onwards, it can be given every $4-6$ hours, but the recommended limit decreases to $600 \mathrm{mg}$.

\section{Tapentadol extended release for the treatment of moderate to severe chronic pain}

Chronic pain is a challenging, widespread problem. Pain management and primary care physicians are often faced with nonmalignant pain syndromes and with those suffering from neuropathic pain. While potent opioids are associated with significant adverse events, nonopioid analgesics may not be sufficient to achieve adequate analgesia among patients with moderate to severe chronic pain. Tapentadol might be effective in patients suffering from chronic somatic and neuropathic pain, secondary to its ability to provide opioid and alpha-adrenergic-mediated analgesia. ${ }^{24}$ In addition, the promising clinical effectiveness and tolerability of tapentadol IR for acute pain prompted the development of an ER preparation (tapentadol ER) for patients suffering from moderate-to-severe chronic pain.

\section{Tapentadol ER Indications}

Longer-acting ER analgesic formulations provide uniformity of effect for patients with chronic pain, as well as improved compliance and convenience for the patient. Several factors influence the decision to use ER opioids, and these include the specific pain diagnosis, expected duration of pain, the severity of the pain, and whether or not the patient has a history of drug abuse or diversion. Tapentadol ER is an ER preparation that is tamper resistant, providing up to 12 hours of pain relief for patients presenting with moderate to severe chronic pain. Tapentadol ER, in clinical trials, was found to be safe and effective for patients with neuropathic, somatic, and mixed pain syndromes (Table 2).
Nociceptive pain, which occurs as a result of direct tissue or neuronal injury, responds well to opioids, and occurs in conditions such as mechanical lower back pain. Injury to nerve fibers, with resultant lowering of noxious threshold in the ascending pain pathways as seen in neuropathic pain, occurs in conditions such as diabetic neuropathic pain. Neuropathic pain is not effectively treated with traditional MORs, ${ }^{25}$ since there is a decrease in the expression of MOR in the dorsal horn following nerve injury. ${ }^{26}$ Because of its unique pharmacodynamics and predictable pharmacokinetics, tapentadol may be a useful option for patients who have failed other medication trials or who are afflicted with treatment emergent adverse events (TEAEs) associated with chronic opioid use.

\section{Chronic somatic pain}

In two separate studies evaluating patients with severe osteoarthritic knee pain and lower back pain, tapentadol ER was found to be more effective than placebo and as effective as oxycodone controlled release (CR). ${ }^{27}$

Tapentadol ER 100-250 mg twice a day (BID) was compared to oxycodone ER 20-50 mg BID. Tapentadol ER was found to effectively relieve moderate to severe chronic low back pain over 15 weeks (comparable to OxyContin, superior to placebo). Tapentadol ER was associated with better GI tolerability than oxycodone CR. The long-term tolerability and safety profile of tapentadol ER was studied by Wild et $\mathrm{al}^{28}$ in chronic low back pain or in chronic knee or hip osteoarthritis as a randomized open label Phase III study. It was found that patients treated with tapentadol ER 100-250 mg BID experienced less nausea, vomiting, constipation, and pruritus than those treated with oxycodone CR (20-50 mg) BID, and gave long-term sustainable pain relief for a period of 1 year. ${ }^{28}$ The key finding in both trials was that tapentadol ER was associated with significantly fewer GI adverse effects; this improvement in GI tolerability was primarily responsible for the lower drug discontinuation rate observed for patients randomized to tapentadol ER compared to oxycodone CR. ${ }^{29}$

\section{Chronic diabetic peripheral neuropathy (DPN)}

Tapentadol ER was studied in the management of severe chronic diabetic neuropathic pain, and was shown to be efficacious and well tolerated compared to placebo. ${ }^{30}$ A Phase III placebo controlled randomized withdrawal study by Schwartz et al studied the efficacy and safety of tapentadol over placebo for the relief of painful diabetic peripheral neuropathy (DPN). In this study, there was a statistically significant improvement of pain relief in patients suffering from painful DPN as compared with placebo with good tolerance. 
There was another clinical trial as well, which evaluated the efficacy, tolerability, and safety of orally administered tapentadol ER. Tapentadol at doses of $100 \mathrm{mg}$ to $250 \mathrm{mg}$ BID was compared with placebo in patients with moderate to severe pain due to chronic, painful DPN who tolerated tapentadol ER after having had an initial treatment. During initial treatment, these patients with DPN had pain improvement after a 3-week, open-label titration period (ClinicalTrials.gov identifier: NCT01041859). This second study was successful in showing the efficacy, safety, and tolerability of tapentadol ER in the treatment of chronic painful DPN. This has led to additional FDA approval for the treatment of neuropathic pain associated with DPN in April 2012. As of this writing, it is the only opioid with such an indication.

Classic opiates are currently considered second-line therapy for neuropathic pain because they are less effective, but medications that have combined mechanisms of action, especially those with NE reuptake inhibition, are effective and are considered first-line therapies. ${ }^{6}$ Studies have shown that enhanced monoamine transmission affects the analgesia of chronic pain, with NE affecting it more than serotonin; tapentadol's antiallodynic and antihyperalgesic properties allow it to be an effective adjunct in the treatment of neuropathic pain. When used in conjunction with current "antineuropathic" medications such as LYRICA ${ }^{\circledR}$ (Pfizer, Inc., New York, NY, USA) and Neurontin ${ }^{\circledR}$ (Pfizer, Inc.), tapentadol may prove to be helpful in patients suffering from chronic neuropathic pain. This could have utility in fibromyalgia, DPN, orthopedic cases involving neuronal damage, amputations, burn patients, patients suffering from chronic low back pain, sciatica, and cancer-related pain syndromes.

\section{Tapentadol ER in patients intolerant of traditional opioids}

Tapentadol ER may be beneficial for any patients currently on opioid treatment for their moderate to severe pain, but who are suffering from their TEAEs. In addition, tapentadol's broad-spectrum antinociception may play a particular role in those suffering from neuropathic pain. When combining these two considerations, the patient population that may benefit from this medication the most is the patient group suffering from cancer-related pain. ${ }^{31}$ A recent prospective study investigated the efficacy and tolerability of tapentadol in the management of 50 opioids-naïve cancer patients with moderate to severe pain and found that it was both well tolerated and efficacious. ${ }^{32}$

This specific population is usually already on several other medications, frequently suffers from GI adverse effects (both associated with the disease and polypharmacy), ${ }^{33}$ and also frequently experiences neuropathic pain from their ailment. However, it should be noted that another vast patient population that has been shown to benefit from tapentadol is the US workforce. Pain conditions have been a common and incredibly debilitating problem in the US workforce, and the estimated cost in lost productivity caused by chronic pain is at $\$ 61$ million; ${ }^{4}$ however, at-work pain alone may not be the sole cause of this loss in productivity. TEAEs of the opioid medications used to treat these pain conditions also contribute greatly to this rising cost. Tapentadol ER was shown to be superior to both placebo and oxycodone CR in improving at-work productivity for patients with osteoarthritic pain. This difference is attributed to the fact that tapentadol ER was able to offer comparable pain control with fewer TEAEs, and therefore exhibiting a lower attrition rate. ${ }^{34}$

\section{Dosing of tapentadol ER}

Tapentadol ER was approved for use in 2011 and has a 12-hour duration of effect and allows for BID dosing. The improvement in GI tolerability, efficacy in treating neuropathic pain, active primary molecule, and convenient dosing suggest that this preparation may offer a safe and effective option for chronic pain management. It is indicated for the management of moderate to severe chronic pain in adults and also for the relief of painful DPN in adults. It is a long-acting preparation approved for use when around the clock pain medication for an extended period of time is required.

The ER form should initially be started at $50 \mathrm{mg}$ every 12 hours with a maximum dose of $500 \mathrm{mg}$ per day. ${ }^{24}$ The ER form is intended for BID dosing. This is intended for chronic pain relief and an IR form for acute pain. If patients have to be converted from tapentadol IR to tapentadol ER, the conversion between the two forms can be achieved quite simply; it is recommended that the patient receive half of the IR requirement on a daily basis as an ER form every 12 hours. The provider should start the patient at a low dose and titrate up towards pain relief. This is done because it is safer to undertreat and provide rescue with IR opioids than to over-treat and subsequently have to manage potential TEAEs. Note that crushing of the ER form could cause IR of the drug and can result in life-threatening events. It should not be used with alcohol as this can cause increased release of the ER form.

The tapentadol ER dosage does not need to be changed because of mild to moderate renal impairment or mild hepatic dysfunction. It has not been studied in patients with severe renal failure (creatinine $<30 \mathrm{~mL} /$ minute) or severe 
hepatic dysfunction (Child-Pugh score $>9$ ). It is therefore not recommended in severe renal or hepatic dysfunction. Tapentadol ER can be used in patients who need pain medication around the clock, and who do not need it as a rescue medication. ${ }^{35}$ Patients do not appear to become tolerant to tapentadol as they do with other opiates. Studies have shown that patients using tapentadol for 24 months have shown no increase in their tapentadol dose. ${ }^{22}$

\section{Cost of use with tapentadol ER}

Tapentadol ER is no more expensive than oxycodone CR or oxymorphone ER, but is significantly more expensive than morphine CR. It may be a first-line drug for patients with GI intolerability or chronic neuropathic complaints.

\section{Interactions of tapentadol IR and tapentadol ER}

An important factor that can determine the tolerability and effectiveness of a new analgesic is whether it interacts with or alters the pharmacokinetics of other medications taken by the patient. Tapentadol would not be expected to have significant drug-drug interactions, as is not metabolized by the hepatic microsomal system and does not inhibit or induce cytochrome p450 enzymes. ${ }^{35}$ When evaluated with patients' coadministered analgesics such as acetaminophen, naproxen, and acetylsalicylic acid, no clinically relevant changes in serum concentrations were noted and no dose readjustment was required.

\section{Common patient considerations for both tapentadol IR and tapentadol ER CNS effects}

Tapentadol IR and tapentadol ER both have similar central nervous system (CNS)-related adverse events as oxycodone. The most common adverse events noted in short- and long-term trials included headache, somnolence, dizziness, and fatigue. ${ }^{24}$ It has been shown that tapentadol IR and ER and oxycodone produce somnolence at a similar rate. ${ }^{36}$ It appears, however, that most patients accommodate to these side effects within 2 weeks of initiation of the drug. Like other opiates, patients should be cautioned when using tapentadol prior to any activity that requires mental attention.

\section{Contraindications}

As with other opioids, tapentadol IR should not be used in patients with respiratory depression. It must be remembered that tapentadol IR is contraindicated in paralytic ileus and in patients who have been taking monoamine oxidase inhibitors (MAOIs) for the last 14 days in order to avoid the additive effects of NE. It must be remembered that tapentadol is a weak serotonin reuptake inhibitor, so it is important to evaluate a potential serotonin syndrome, although it is a rare event and patients in clinical trials were allowed to continue stable doses of selective serotonin reuptake inhibitors (SSRIs). Interactive effects with other pain adjuvants and powerful drugs such as opioids, anesthetics, hypnotics, sedatives, alcohol, and other CNS depressants should always be considered.

\section{Tolerability and safety}

Tapentadol has a more favorable side effect profile compared to other opiates because of its reduced affinity for the mu receptor. ${ }^{36}$ Tapentadol has been shown to have a similar incidence of non-GI side effects as oxycodone and morphine, but it has a less severe side effect profile. ${ }^{37}$ Pruritus is an annoying opioid-related adverse effect. In bunionectomy trials 1 and 2, patients treated with tapentadol experienced less pruritus when compared with oxycodone IR; however, these differences did not reach statistical significance as seen in bunionectomy trial $2 .^{38}$

The most common side effects of tapentadol are nausea (30\%), dizziness (24\%), vomiting (18\%), and somnolence $(15 \%) .{ }^{8}$ Higher satisfaction rates seen during the studies led to increased tolerability and lower discontinuation rates among the patients.

\section{Improved gastrointestinal tolerability}

The GI tolerability of tapentadol IR has been compared to oxycodone in two surgical trials and one long-term trial. Patients in Bunionectomy Trial 2 who received tapentadol IR $50 \mathrm{mg}$ and $75 \mathrm{mg}$ reported less nausea/vomiting when compared with oxycodone IR $10 \mathrm{mg} .{ }^{22}$ In a long-term 90-day trial evaluating patients with lower back pain or osteoarthritis of the knee, those treated with tapentadol reported less nausea, vomiting, and constipation when compared to those treated with oxycodone. Fewer patients in the tapentadol IR group discontinued therapy because of these adverse effects. It is conceivable that the noradrenergic analgesic effect of tapentadol adds to the analgesia, while having less impact on GI motility. In a 90-day study, it was shown that tapentadol IR was had similar pain relief as compared to oxycodone IR, with improved GI tolerability as compared with oxycodone IR. ${ }^{39}$ 
Table I Indications for tapentadol IR

Gl intolerance

Nausea and vomiting to opioids

Opioid related itching

Moderate to severe acute pain

Abbreviations: IR, immediate release; GI, gastrointestinal.

In a clinical trial by Hartrick et $a l,{ }^{40}$ in patients with uncontrolled osteoarthritis pain awaiting joint replacement surgery there was lower nausea and vomiting, and higher satisfaction with lower discontinuation rates versus oxycodone IR.

Constipation secondary to opiate use is particularly bothersome in chronic pain patients. Consequently, stool softeners and/or laxatives need to be added to a daily medication regime of people taking these medications. However, in half of these patients, it was seen that even stool softeners and/or laxatives do not alleviate the problem. Multiple studies have shown that tapentadol ER has a $60 \%$ reduction in constipation when compared to oxycodone. ${ }^{32}$

Tapentadol IR and tapentadol ER may be a good alternative to traditional opioids in patients who are prone to its adverse GI effects (Table 1). It may be able to provide effective pain control without sacrificing GI comfort. Interestingly, while it appears that tapentadol IR is better tolerated, a study has shown that when compared to equipotent doses of oxycodone, both drugs delay gastric emptying as compared to placebo, and they have no effect on colonic transit time. ${ }^{41}$ Despite these advantages, like all opioids, tapentadol is strictly contraindicated in

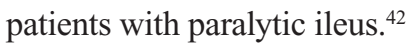

\section{Respiratory depression}

Respiratory depression is a concern with all opioids. Likewise, tapentadol IR and tapentadol ER are contraindicated in patients with severe asthma in unmonitored settings without accessible resuscitative equipment. ${ }^{22}$

\section{Less concern of tolerance and withdrawal}

One of the main reasons that pain is undertreated is the fear of the development of tolerance, dependence,

Table 2 Indications for tapentadol ER

Gl intolerance

Constipation

Patients with diabetic neuropathy

Moderate to severe chronic pain

Abbreviations: ER, extended release; GI, gastrointestinal. and subsequent withdrawal. ${ }^{43}$ Opioid withdrawal and dependence may be associated with the lowering of noradrenergic tone from the locus coeruleus. ${ }^{44}$ This then causes an increase in the noradrenergic activity of the locus coeruleus. ${ }^{45}$ Patients treated long term with tapentadol IR and ER were less likely to experience opioid withdrawal symptoms following abrupt discontinuation than those treated with oxycodone (17\% versus $29 \%$ ). This reduction in symptoms may be related to the lower binding affinity of tapentadol at the MOR. Similar to traditional agonists, the risk of precipitating opioid withdrawal symptoms increases following abrupt discontinuation, for this reason tapentadol should always be slowly tapered over several days to a week. It should always be recognized that tapentadol is a powerful analgesic that has significant abuse and dependence potential.

\section{Less electrophysiologic and hemodynamic concerns}

Torsades de pointes is a type of polymorphic ventricular tachycardia associated with QT prolongation, and is the most common drug-induced arrhythmia. Opioid analgesics, such as methadone and buprenorphine, have been associated with QT prolongation even at doses less than $80 \mathrm{mg} .{ }^{46}$ It is therefore important to note that there is no evidence that tapentadol prolongs the QT interval at therapeutic or supratherapeutic levels. ${ }^{47}$ This suggests that tapentadol may be given to patients with preexisting QT prolongation with less concern that it would precipitate torsades de pointes. Long-term administration of tapentadol IR and ER was found to be comparable to both placebo and an opioid comparator in maintaining stable blood pressure and heart rate.

\section{Peripartum concerns}

The safety and effectiveness of tapentadol has not been evaluated in patients who are pregnant or who are breastfeeding, and is listed as a class C drug by the FDA. ${ }^{23}$

\section{Contraindications}

Like most opioid analgesics, tapentadol is contraindicated in patients who have taken MAOIs within the last 14 days due to the potential synergistic effects on NE levels, which may precipitate cardiac events. This combination may also elevate the level of serotonin. Tapentadol is a fairly selective inhibitor of NE and has minimal effects on serotonin reuptake. Nevertheless, prescribers must exercise caution coprescribing 
tapentadol for patients taking SSRIs and triptans. The additive effects on serotonin levels may cause the life-threatening serotonin syndrome. While this is a relatively rare event, caregivers should monitor warning signs such as mental status changes, autonomic instability, and neuromuscular abnormalities. ${ }^{39}$ It should be noted that patients on stable doses of SSRIs and serotonin-norepinephrine reuptake inhibitors were enrolled in all clinical trials, and no evidence of serotonin syndrome was observed in patients randomized to receive long-term treatment with tapentadol.

Tapentadol IR and tapentadol ER are not recommended in severe renal or hepatic impairment or in circulatory shock since they could further reduce blood pressure by vasodilatation. Caution has to be exercised with the use of tapentadol IR and tapentadol ER in the presence of other CNS depressants such as muscle relaxants, neuroleptics, illicit drugs, anxiolytics, opioids, and sedatives.

\section{Conclusion}

Tapentadol is a novel and potentially useful analgesic drug for the treatment of moderate to severe acute and chronic pain. Unlike comparable options, its mechanism of action is purely inhibitory to both the ascending and descending pain pathways; it is not a racemic compound and has no active metabolites. In acute moderate to severe pain, it has an analgesic effect that is comparable to oxycodone IR. One of the great advantages in the treatment of moderate to severe acute pain is improved GI tolerability. Its efficacy for chronic pain is similar to that of traditional opioids, but it has an improved side effect profile, which could contribute to improved compliance. Tapentadol ER could be effective in a wide spectrum of patients with moderate to severe chronic pain such as osteoarthritic pain, DNP, and cancer pain. It may also prove to be an effective option for those dealing with pain at work. Although more clinical data is needed on its long-term efficacy in various populations and potential for abuse and toxicity, its excellent safety profile and patients' high levels of satisfaction compared to mu opioids in long-term acute and chronic pain trials make tapentadol an exciting addition to the therapeutic armamentarium in pain management.

\section{Disclosure}

The authors report no conflicts of interest in this work.

\section{References}

1. Hutchison RW. Challenges in acute post-operative pain management. Am J Health Syst Pharm. 2007;64(6 Suppl 4):S2-S5.
2. Apfelbaum JL, Chen C, Mehta SS, Gan TJ. Postoperative pain experience: results from a national survey suggest postoperative pain continues to be undermanaged. Anesth Analg. 2003;97(2):534-540, table of contents.

3. Woolf CJ; for American College of Physicians; American Physiological Society. Pain: moving from symptom control toward mechanismspecific pharmacologic management. Ann Intern Med. 2004;140(6): $441-451$.

4. Kehlet H. Multimodal approach to control postoperative pathophysiology and rehabilitation. Br J Anaesth. 1997;78(5):606-617.

5. Wisconsin Medical Society Task Force on Pain Management. Guidelines for the assessment and management of chronic pain. WMJ. 2004;103(3):13-42.

6. Moore RA, McQuay HJ. Prevalence of opioid adverse events in chronic non-malignant pain: systematic review of randomised trials of oral opioids. Arthritis Res Ther. 2005;7(5):R1046-R1051.

7. Cherny NI. Opioid analgesics: comparative features and prescribing guidelines. Drugs. 1996;51(5):713-737.

8. Crain SM, Shen KF. Antagonists of excitatory opioid receptor functions enhance morphine's analgesic potency and attenuate opioid tolerance/ dependence liability. Pain. 2000;84(2-3):121-131.

9. Leslie JB. Alvimopan: a peripherally acting mu-opioid receptor antagonist. Drugs Today (Barc). 2007;43(9):611-625.

10. Young A, Buvanendran A. Recent advances in multimodal analgesia. Anesthesiology Clin. 2012;30(1):91-100.

11. Tzschentke TM, Christoph T, Kögel B, et al. (-)-(1R,2R)-3(3-dimethylamino-1-ethyl-2-methyl-propyl)-phenol hydrochloride (tapentadol $\mathrm{HCl}$ ): a novel mu-opioid receptor agonist/norepinephrine reuptake inhibitor with broad-spectrum analgesic properties. J Pharmacol Exp Ther. 2007;323(1):265-276.

12. Vadivelu N, Timchenko A, Huang Y, Sinatra R. Tapentadol extendedrelease for treatment of chronic pain: a review. J Pain Res. 2011;4: 211-218.

13. Wade WE, Spruill WJ. Tapentadol hydrochloride: a centrally acting oral analgesic. Clin Ther. 2009;31(12):2804-2818.

14. Raffa RB, Buschmann H, Christoph T, et al. Mechanistic and functional differentiation of tapentadol and tramadol. Expert Opin Pharmacother. 2012;13(10):1437-1449.

15. Kress HG. Tapentadol and its two mechanisms of action: is there a new pharmacological class of centrally-acting analgesics on the horizon? Eur J Pain. 2010;14(8):781-783.

16. Staiger TO, Gaster B, Sullivan MD, Deyo RA. Systematic review of antidepressants in the treatment of chronic low back pain. Spine (Phila Pa 1976). 2003;28(22):2540-2545.

17. Lagas JS, Wagenaar JF, Huitema AD, et al. Lethal morphine intoxication in a patient with a sickle cell crisis and renal impairment: case report and a review of the literature. Hum Exp Toxicol. 2011;30(9):1399-1403.

18. Terlinden R, Kogel BY, Englberger W, Tzschentke TM. In vitro and in vivo characterization of tapentadol metabolites. Methods Find Exp Clin Pharmacol. 2010;32(1):31-38.

19. Erlich DR, Bodine W. Tapentadol (nucynta) for treatment of pain. Am Fam Physician. 2012;85(9):910-911.

20. Obradovic M, Ikenberg R, Hertel N, Antoñanzas F, Gálvez R, Liedgens H. Cost-effectiveness of tapentadol in severe chronic pain in Spain: a cost analysis of data from RCTs. Clin Ther. 2012;34(4):926-943.

21. Daniels SE, Upmalis D, Okamoto A, Lange C, Häeussler J. A randomized, double-blind, phase III study comparing multiple doses of tapentadol IR, oxycodone IR, and placebo for postoperative (bunionectomy) pain. Curr Med Res Opin. 2009;25(3):765-776.

22. Daniels S, Casson E, Stegmann JU, et al. A randomized, double-blind, placebo-controlled phase 3 study of the relative efficacy and tolerability of tapentadol IR and oxycodone IR for acute pain. Curr Med Res Opin. 2009;25(6):1551-1561.

23. Stegmann JU, Weber H, Steup A, Okamoto A, Upmalis D, Daniels S. The efficacy and tolerability of multiple-dose tapentadol immediate release for the relief of acute pain following orthopedic (bunionectomy) surgery. Curr Med Res Opin. 2008;24(11):3185-3196. 
24. Schröder W, Vry JD, Tzschentke TM, Jahnel U, Christoph T. Differential contribution of opioid and noradrenergic mechanisms of tapentadol in rat models of nociceptive and neuropathic pain. Eur $J$ Pain. 2010;14(8):814-821.

25. Nicholson B. Differential diagnosis: nociceptive and neuropathic pain. Am J Manag Care. 2006;12(Supp1 9):S256-S262.

26. Millan MJ. Descending control of pain. Prog Neurobiol. 2002;66(6): $355-474$

27. Buynak R, Shapiro DY, Okamoto A, et al. Efficacy and safety of tapentadol extended release for the management of chronic low back pain: results of a prospective, randomized, double-blind, placeboand active-controlled Phase III study. Expert Opin Pharmacother. 2010;11(11):1787-1804

28. Wild JE, Grond S, Kuperwasser B, et al. Long-term safety and tolerability of tapentadol extended release for the management of chronic low back pain or osteoarthritis pain. Pain Pract. 2010;10(5):416-427.

29. Afilalo M, Etropolski MS, Kuperwasser B, et al. Efficacy and safety of Tapentadol extended release compared with oxycodone controlled release for the management of moderate to severe chronic pain related to osteoarthritis of the knee: a randomized, double-blind, placebo- and active-controlled phase III study. Clin Drug Investig. 2010;30(8):489-505.

30. Schwartz S, Etropolski M, Shapiro DY, et al. Safety and efficacy of tapentadol ER in patients with painful diabetic peripheral neuropathy: results of a randomized-withdrawal, placebo-controlled trial. Curr Med Res Opin. 2011;27(1):151-162.

31. Weber H, Stegmann J, Upmalis D. Tapentadol immediate release for the relief of acute pain following orthopedic surgery of the forefoot: Efficacy and tolerability results from a randomized, double blind, phase II study [Abstract]. Eur J Anaesthesiol. 2008;25(Suppl 44): 14AP11-14AP13.

32. Mercadante S, Porzio G, Ferrera P, et al. Tapentadol in cancer pain management: a prospective open-label study. Curr Med Res Opin. 2012;28(11):1775-1779.

33. Baines MJ. ABC of palliative care. Nausea, vomiting, and intestinal obstruction. BMJ. 1997;315(7116):1148-1150.

34. Lerner D, Chang H, Rogers WH, et al. Imputing at-work productivity loss using results of a randomized controlled trial comparing tapentadol extended release and oxycodone controlled release for osteoarthritis pain. J Occup Environ Med. 2012;54(8):933-938.

35. Kneip C, Terlinden R, Beier H, Chen G. Investigations into the drugdrug interaction potential of tapentadol in human liver microsomes and fresh human hepatocytes. Drug Metab Lett. 2008;2(1):67-75.
36. Steigerwald I, Müller M, Kujawa J, Balblanc JC, Calvo-Alén J. Effectiveness and safety of tapentadol prolonged release with tapentadol immediate release on-demand for the management of severe, chronic osteoarthritis-related knee pain: results of an open-label, phase $3 \mathrm{~b}$ study. J Pain Res. 2012;5:121-138.

37. Hartrick CT, Rodríguez Hernandez JR. Tapentadol for pain: a treatment evaluation. Expert Opin Pharmacother. 2012;13(2):283-286.

38. Daniels SE, Upmalis D, Okamoto A, Lange C, Häeussler J. A randomized, double-blind, phase III study comparing multiple doses of tapentadol IR, oxycodone IR, and placebo for postoperative (bunionectomy) pain. Curr Med Res Opin. 2009;25(3):765-776.

39. Hale M, Upmalis D, Okamoto A, Lange C, Rauschkolb C. Tolerability of tapentadol immediate release in patients with lower back pain or osteoarthritis of the hip or knee over 90 days: a randomized, doubleblind study. Curr Med Res Opin. 2009;25(5):1095-1104.

40. Hartrick C, Van Hove I, Stegmann JU, Oh C, Upmalis D. Efficacy and tolerability of tapentadol immediate release and oxycodone $\mathrm{HCl}$ immediate release in patients awaiting primary joint replacement surgery for end-stage joint disease: a 10-day, phase III, randomized, double-blind, active- and placebo-controlled study. Clin Ther. 2009;31(2):260-271.

41. Jeong ID, Camilleri M, Shin A, et al. A randomised, placebo-controlled trial comparing the effects of tapentadol and oxycodone on gastrointestinal and colonic transit in healthy humans. Aliment Pharmacol Ther. Epub February 21, 2012.

42. Froicu D, Sinatra RS. Tapentadol. In: Sinatra RS, Jahr JS, Watkins-Pitchford JM, editors. The Essence of Analgesia and Analgesics. Cambridge, UK: Cambridge University Press; 2010: 143-147.

43. Friedman DP. Perspectives on the medical use of drugs of abuse. J Pain Symptom Manage. 1990;5(Suppl 1):S2-S5.

44. Cruz HG, Berton F, Sollini M, et al. Absence and rescue of morphine withdrawal in GIRK/Kir3 knock-out mice. J Neurosci. 2008;28(15): 4069-4077.

45. Aghajanian GK. Tolerance of locus coeruleus neurones to morphine and suppression of withdrawal response by clonidine. Nature. 1978;276(5684):186-188.

46. Huh B, Park CH. Retrospective analysis of low-dose methadone and QTc prolongation in chronic pain patients. Korean J Anesthesiol. 2010;58(4):338-343.

47. Oh C, Rengelshausen J, Mangold B, et al. A thorough QT/QTc study of multiple doses of tapentadol immediate release in healthy subjects. Int J Clin Pharmacol Ther. 2010;48(10):678-687.
Drug, Healthcare and Patient Safety

\section{Publish your work in this journal}

Drug, Healthcare and Patient Safety is an international, peer-reviewed open-access journal exploring patient safety issues in the healthcare continuum from diagnostic and screening interventions through to treatment, drug therapy and surgery. The journal is characterized by the rapid reporting of reviews, original research, clinical, epidemiological and

\section{Dovepress}

post-marketing surveillance studies, risk management, health literacy and educational programs across all areas of healthcare delivery. The manuscript management system is completely online and includes a very quick and fair peer-review system. Visit http://www.dovepress.com/ testimonials.php to read real quotes from published authors. 